\title{
Change of Platelet Reactivity to Antiplatelet Therapy after Stenting Procedure for Cerebral Artery Stenosis: VerifyNow Antiplatelet Assay before and after Stenting
}

Deok Hee Lee, MD', Ho Sung Kim, MD', Sun Mi Kim, MD², Sun-Uck Kwon, MD², Dae Chul Suh, MD

Purpose: VerifyNow antiplatelet assays were performed before and after stenting for various cerebral artery stenoses to determine the effect of the procedure itself to the function of dual antiplatelets given.

Materials and Methods: A total of 30 consecutive patients underwent cerebral arterial stenting procedure were enrolled. The antiplatelet pretreatment regimen was aspirin (100 mg daily) and clopidogrel (300 mg of loading dose followed by $75 \mathrm{mg}$ daily). VerifyNow antiplatelet assay performed before and right after stenting. The two test results were compared in terms of aspirin-reaction unit (ARU), P2Y12 reaction units (PRU), baseline (BASE), and percentage inhibition. We evaluated occurrence of any intra-procedural in-stent thrombosis or immediate thromboembolic complication, and ischemic events in 1-month follow-up.

Results: The median Pre-ARU was 418 (range, 350-586). For clopidogrel the medians of the pre-BASE, PRU, and percent inhibition were 338 (279-454), 256 (56-325), and 27\% (0-57\%). The medians of the post-ARU, BASE, PRU, and percent inhibition after stenting were 469 (range, 389-573), 378 $(288-453), 274(81-370)$, and $26 \%(0-79 \%)$. There was a significant increase of ARU $(p=0.045)$, $\operatorname{BASE}(p=0.026)$, and PRU $(p=0.018)$ before and after stenting. One immediate thromboembolic event was observed in poor-response group after stenting. There was no in-stent thrombosis and ischemic event in 1-month follow-up.

Conclusion: We observed a significant increase of platelet reactivity to dual antiplatelet therapy right after stenting procedure for various cerebral arterial stenoses.

Key Words : Stent; Cerebrovascular disorders; Atherosclerosis; Antiplatelet drugs;

VerifyNow antiplatelet assay

\footnotetext{
This study was supported by a grant (A102065) from the Korea Health 21 Research and Development Project, Ministry of Health, Welfare and Family Affairs, Republic of Korea.

Departments of ${ }^{1}$ Radiology and Research Institute of Radiology, ${ }^{3}$ Neurology, University of Ulsan, College of Medicine, Asan Medical Center, Seoul, Korea

'2Department of Radiology, Kyung Hee University Hospital at Gangdong, Seoul, Korea

Received August 22, 2011; accepted after revision November 7, 2011.

Correspondence to: Deok Hee Lee, MD, PhD, Department of Radiology, Asan Medical Center, 88 Olympic-ro 43-gil, Songpa-gu, Seoul 138736, Korea.

Tel. 82.2.3010.5944 Fax.82.2.476.0090 E-mail: dhlee2000@gmail.com

This is an Open Access article distributed under the terms of the Creative Commons Attribution Non-Commercial License (http://creativecommons.org/licenses/by-nc/3.0) which permits unrestricted non-commercial use, distribution, and reproduction in any medium, provided the original work is properly cited.
} 


\section{Deok Hee Lee, et al.}

Dual-antiplatelet treatment has become a standard pretreatment medication for cerebrovascular stenting procedures such as carotid/vertebral artery stenting or intracranial artery stenting. Since the retraction of ticlopidine, oral administration of aspirin and clopidogrel is the standard regimen in most of the cases [1-3]. However, recently there has been growing concern about poor biochemical resistance to the antiplatelet therapy especially to clopidogrel in our neurovascular arena and among our cardiology colleagues as well [4-7].

Owing to the recent advent of a point-of-care assay for the antiplatelet monitoring, such as VerifyNow Assay (Accumetrics, San Diego, CA, U.S.A.), it has become quite easy and convenient to monitor antiplatelet function of both aspirin and clopidogrel $[6$, $8,9]$. According to our recent experience, poor response rate to clopidogrel was quite high in our neurovascular patients group as well [6]. However, in the generalization and interpretation of the test result, e.g., percent inhibition (\% inhibition) and P2Y 12 reaction unit (PRU), we found there was no solid cutoff values of poor response. Each paper reports its own definition and result [10-14].

There have been several possible suggestions on the possible etiology of poor response to clopidogrel [5, 15]. In addition to the individual variability of clopidogrel response, we hypothesized that the measurement result could be varied by the timing of measurement. Although we are focusing on the pre-stenting value of the test in most of the cases, some are focusing on the post-procedural test result for the prediction of longterm effect of the clopidogrel treatment [16].

In this study we focused on the possible influence of stenting procedure itself to the test result. We hypothesized that the test result could be increased by intraarterial procedure itself since the intravascular manipulation using various intravascular devices and the remained stent mesh in the arterial lumen might increase platelet reactivity.

\section{MATERIALS AND METHODS}

\section{Patients}

After obtaining an approval of our institutional review board, we prospectively enrolled patients who underwent elective supraaortic and/or cerebrovascular stenting procedures due to atherosclerotic stenosis of the internal carotid artery (ICA), subclavian artery, vertebral artery, or intracranial arteries from October 2008 to September 2009. Exclusion criteria were insufficient antiplatelet pre-treatment before the procedure. There was no control group in this study.

Our definition of sufficient antiplatelet pretreatment was more than 5 days of dual antiplatelet medication with daily doses of $100 \mathrm{mg}$ of aspirin and $75 \mathrm{mg}$ of clopidogrel following loading doses of 300-500 mg of aspirin and $300 \mathrm{mg}$ of clopidogrel.

\section{Procedure and antiplatelet function assay}

We double checked whether the patient had his/her morning dose of antiplatelets before the procedure. Immediately after insertion of the femoral artery sheath, $9 \mathrm{ml}$ of arterial blood was drawn to measure baseline activated clotting time (ACT) and preprocedural VerifyNow Aspirin and P2Y12 Assays (Accumetrics, San Diego, CA. U.S.A.), which were pre-procedural aspirin reaction unit (Pre-ARU) for aspirin, and pre-procedural base value (Pre-BASE), pre-procedural P2Y12 reaction unit (Pre-PRU), and pre-procedural percent inhibition (Pre-\%inhibition) for clopidogrel. Technical details of the assay were already described before [6].

Stenting procedure was performed after systemic heparinization and the target ACT value was 250-300 sec. We waited 15 to 20 minutes after completion of the stenting to check any occurrence of acute in-stent thrombosis or distal thromboembolism. Right before the removal of the femoral sheath, another $9 \mathrm{ml}$ of arterial blood was drawn to measure post-procedural ACT, Post-ARU, Post-BASE, Post-PRU, and Post$\%$ inhibition. We did not give any additive dosage of antiplatelet according to the test result unless there was any thrombotic complication during or after the procedure since we did not have any solid evidence of proper platelet function inhibition for the neurovascular stenting procedure yet.

\section{Post-procedural care and Analysis}

After the procedure the patient was sent to neurological intensive care unit. We carefully monitored the patient for the occurrence of any neurological change. If the patient was stable for 24 hours we transferred the patient to general ward. Any occurrence of clinical event was noticed for one month afterwards.

Statistical analysis was performed using SPSS (SPSS, Inc., Chicago, IL, U.S.A.). We summarized the two test results, pre- and post-procedural values of the antiplatelet function assay by presenting medians and their ranges or quartile ranges of ARU, PRU, BASE, and $\%$ inhibition. We compared the pre- and postprocedural values of the assay with use of Wilcoxon signed-rank test. 


\section{RESULTS}

A total of 30 patients were enrolled in this study. There were 23 men and 7 women with their mean age of 62.6 years. The target lesion location was the proximal ICA in 12, distal ICA in 2, middle cerebral artery in 6 , subclavian artery in 2 , and vertebrobasilar system in 8.

The median Pre-ARU was 418 (range, 350-586). For clopidogrel the medians of the pre-BASE, PRU, and $\%$ inhibition were 338 (range, 279-454), 256 (range, 56-325), and 27\% (range, 0-57\%). After stenting, the medians of the post-ARU, BASE, PRU, and $\%$ inhibition were 469 (range, 389-573), 378 (range, 288-453), 274 (range, 81-370), and 26\% (range $0-79 \%$ ). There were significant changes of $\operatorname{ARU}(\mathrm{p}=0.045), \operatorname{BASE}(\mathrm{p}=0.026)$, and PRU $(\mathrm{p}=$ 0.018 ) before and after stenting (Fig. 1). Since the BASEs and PRUs increased at the same time, there was no significant difference in percent inhibitions.

There was no acute in-stent thrombosis or distal thromboembolism during the procedure. However, there was a case of delayed symptomatic patient who showed mild facial weakness and dizziness about five hours after the completion of proximal basilar artery stent placement. Diffusion-weighted image obtained thereafter showed acute infarction at lower medulla and cerebellum suggesting stent-associated ischemic lesions. His pre- and post- ARUs, BASEs, PRUs, and $\%$ inhibitions were 418 and 560, 413 and 432, 299 and 270 , and $27 \%$ and $37 \%$. His symptom gradually improved overtime without residual deficit. There was no further ischemic event on 1-month follow-up. There was no further clinical event during the one-month follow-up period.

\section{DISCUSSION}

The importance of dual-antiplatelet pretreatment with use of aspirin and clopidogrel cannot be overemphasized in our neurovascular stenting procedures. Even with strict coverage more than 3 days, we occasionally experience acute or subacute thromboembolic phenomena during or after the procedure. Poor biochemical response to both aspirin and/or clopidogrel has been postulated as the possible cause of poor antiplatelet response [4]. With advent of a convenient point-of-care testing device, we can adjust our antiplatelet regimen on individual basis by administrating double loading doses and/or increased maintenance doses $[6,7,17]$.

With accumulation of our experience, we became realized that poor response to clopidogrel is much more frequent than poor response to aspirin and PRU is more ideal than \% inhibition in terms of poor response to clopidogrel $[6,7,17]$. The question is what are the cutoff values of the test results? To answer the question,

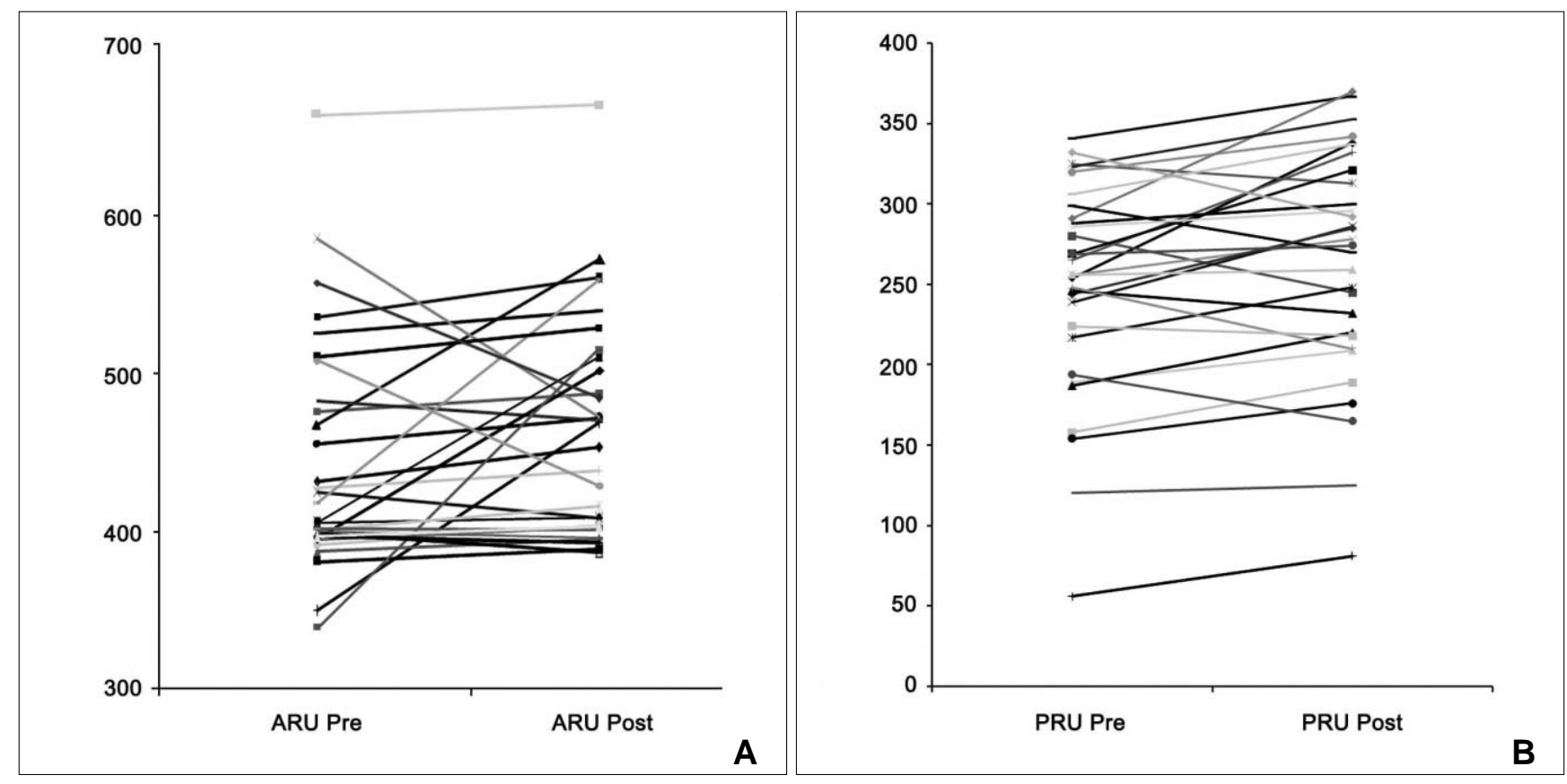

Fig. 1. Individual changes of $A R U(A)$ and $P R U(B)$ values before and after the stenting procedures. 


\section{Deok Hee Lee, et al.}

several studies have been undergone by applying the cut-off values calculated with receiver-operating-curve analysis or 4th quartile range as the high risk group [11, $13,14]$. Although the cut-off values of PRU are around $230-260$, there is quite large variability of the values from report to report.

We believe that each institution should have its own cut-off value for this. In setting up individual internal reference values, consistency in clinical factors should be considered. One of possible influencing factors could be the timing of blood sampling as we observed in this study. The study idea was based on the idea that intravascular manipulation of various interventional devices such as catheters, guidewires, balloon angioplasty catheters, and/or stents and repeat use of iodinated contrast media may influence on the reactivity of partially inhibited platelets due to dualantiplatelet pretreatment. In the interpretation of a certain test result, we can overestimate antiplatelet reactivity if the blood sample was taken after the procedure.

There are several limitations of this study. The number of patient was small. However, we did not feel a strong need of larger patient size since we could observe statistically significant increase of platelet reactivity after procedure with our limited patient cohort. Another limitation was we could not correlate the change with occurrence of any clinical event. However, since there was no case of acute in-stent thrombosis in our case and there was only one patient who was symptomatic after the procedure, it was impossible to draw any conclusion on the clinical significance of our observation. Large patient size could answer this question.

With a point-of-care assay, we observed a significant increase of platelet reactivity to dual antiplatelet therapy after stenting procedure for cerebral arterial stenosis. Consistency in measurement timing should be considered in setting up an institutional internal reference values of the assay.

\section{References}

1. Stenting of symptomatic atherosclerotic lesions in the vertebral or intracranial arteries (SSYLVIA): study results. Stroke 2004;35: 1388-1392

2. Suh DC, Kim SJ, Lee DH, Kim W, Choi CG, Lee JH, et al. Outcome of endovascular treatment in symptomatic intracranial vascular stenosis. Korean J Radiol 2005;6:1-7

3. Fiorella D, Albuquerque FC, Woo H, Rasmussen PA, Masaryk TJ, McDougall CG. Neuroform in-stent stenosis: incidence, natural history, and treatment strategies. Neurosurgery 2006;59: $34-42$
4. Gurbel PA, Bliden KP, Hiatt BL, O’Connor CM. Clopidogrel for coronary stenting: response variability, drug resistance, and the effect of pretreatment platelet reactivity. Circulation 2003; 107:2908-2913

5. Gurbel PA, Tantry US. Aspirin and clopidogrel resistance: Consideration and management. J Interv Cardiol 2006;19:439448

6. Lee DH, Arat A, Morsi H, Shaltoni H, Harris JR, Mawad ME. Dual antiplatelet therapy monitoring for neurointerventional procedures using a point-of-care platelet function test: a singlecenter experience. AJNR Am J Neuroradiol 2008;29:1389-1394

7. Kang HS, Kwon BJ, Kim JE, Han MH. Preinterventional clopidogrel response variability for coil embolization of intracranial aneurysms: clinical implications. AJNR Am J Neuroradiol 2010;31:1206-1210

8. Malinin A, Pokov A, Swaim L, Kotob M, Serebruany V. Validation of a VerifyNow-P2Y12 cartridge for monitoring platelet inhibition with clopidogrel. Methods Find Exp Clin Pharmacol 2006;28:315-322

9. Jakubowski JA, Payne CD, Li YG, Brandt JT, Small DS, Farid NA, et al. The use of the VerifyNow-P2Y12 point-of-care device to monitor platelet function across a range of inhibition levels following prasugrel and clopidogrel administration. Thromb Haemost 2008;99:409-415

10. Malinin A, Pokov A, Spergling M, Defranco A, Schwartz K, Schwartz D, et al. Monitoring platelet inhibition after clopidogrel with the VerifyNow-P2Y12 rapid analyzer: the verify thrombosis risk assessment (VERITAS) study. Thromb Res 2007;119:277284

11. Paniccia R, Antonucci E, Gori AM, Marcucci R, Giglioli C, Antoniucci D, et al. Different methodologies for evaluating the effect of clopidogrel on platelet function in high-risk coronary artery disease patients. J Thromb Haemost 2007;5:1839-1847

12. Jeong YH, Kim IS, Choi BR, Kwak CH, Hwang JY. The optimal threshold of high post-treatment platelet reactivity could be defined by a point-of-care VerifyNow-P2Y12 assay. Eur Heart J 2008;29:2186-2187

13. Price MJ, Endemann S, Gollapudi RR, Valencia R, Stinis CT, Levisay JP, et al. Prognostic significance of post-clopidogrel platelet reactivity assessed by a point-of-care assay on thrombotic events after drug-eluting stent implantation. Eur Heart $J$ 2008;29:992-1000

14. Patti G, Nusca A, Mangiacapra F, Gatto L, D’Ambrosio A, Di Sciascio G. Point-of-care measurement of clopidogrel responsiveness predicts clinical outcome in patients undergoing percutaneous coronary intervention results of the (antiplatelet therapy for reduction of myocardial damage during angioplasty-platelet reactivity predicts outcome) study. $\mathrm{J} \mathrm{Am}$ Coll Cardiol 2008;52:1128-1133

15. Gurbel PA, Tantry US. Drug insight: clopidogrel nonresponsiveness. Nat Clin Pract Cardiovasc Med 2006;3:387-395

16. Trenk D, Hochholzer W, Fromm MF, Chialda LE, Pahl A, Valina $\mathrm{CM}$, et al. Cytochrome and high on-clopidogrel platelet reactivity associated with adverse 1 -year clinical outcome of elective percutaneous coronary intervention with drug-eluting or baremetal stents. J Am Coll Cardiol 2008;51:1925-1934

17. Comin J, Kallmes D. Clopidogrel (plavix). AJNR Am J Neuroradiol 2011;32:2002-2004 